\title{
Splitting and Structure of the Giant Quadrupole Resonances in Deformed Nuclei
}

\author{
Dieter Zawischa \\ Institute of Theoretical Physics, Technical University of Hannover, 3 Hannover, West Germany \\ and \\ Josef Speth \\ Institute of Nuclear Physics, Kernforschungsanlage Jülich, 517 Jülich, West Germany, and \\ Department of Physics, University of Bonn, 53 Bonn, West Germany
}

(Received 15 January 1976)

\begin{abstract}
We have performed microscopic calculations of the low-lying and high-lying $K^{\pi}=0^{+}$, $1^{+}$, and $2^{+}$states in deformed rare-earth nuclei. The centroids of the isoscalar giant quadrupole resonance in ${ }^{160} \mathrm{Dy},{ }^{170} \mathrm{Yb}$, and ${ }^{184} \mathrm{~W}$ are at $11.58,11.44$, and $11.22 \mathrm{MeV}$, respectively. A broadening of these resonances due to the deformation is predicted which varies appreciably in the different nuclei. About $20 \%$ of the monopole strength is found in the same energy range.
\end{abstract}

In the last two years giant resonances of multipolarity different from the well-known dipole resonance have been detected experimentally by inelastic electron and proton scattering. ${ }^{1}$ A review of this field may be found in Satchler. ${ }^{2}$ Until now most of the experimental work has been done on spherical nuclei. The isoscalar giant quadrupole resonance (GQR), e.g., has been found at an excitation energy which agrees qualitatively with the prediction of Bohr and Mottelson. ${ }^{3}$ In the special cases of doubly closed-shell nuclei random-phase-approximation (RPA) calculation $s^{4,5}$ give semiquantitative agreement with the experimental data.

Very recently the GQR has also been found experimentally in deformed rare-earth nuclei..$^{6-8}$ In these experiments the width of the GQR was found to be broader than in the spherical isotopes.
In the case of the giant dipole resonances this is an experimentally well established and also theoretically understood phenomenon. The situation, however, is controversial for the GQR: The dynamic collective model predicts ${ }^{9}$ a splitting into five levels as a result of the deformation. In a schematic calculation using the harmonic-oscillator model and a quadrupole-quadrupole interaction one gets a splitting into three different levels and a broadening of 6 or $2 \mathrm{MeV}$ depending on the quadrupole-quadrupole force used. Neither the transition probability nor the absolute energies are reported in Ref. 8.

In this Letter we present results of a realistic microscopic calculation within the quasiparticleRPA of the low-lying and high-lying $K^{\pi}=0^{+}, 1^{+}$, and $2^{+}$(intrinsic) states. We used data for the low-lying states to check the input data of our 
theory such as single-particle energies, effective particle-hole and particle-particle interactions, and the size of the configuration space. This is a generalization of previous calculations in spherical nuclei. ${ }^{4}$ For the effective interactions density-dependent zero-range forces have been used. The force parameters and the singleparticle wave functions are the same as in a recent analysis of isotope shifts and $K^{\pi}=0^{+}$excitations. ${ }^{10}$ We do not repeat here the well-known formulas of the quasiparticle-RPA theory, which may be found in Ref. 10. We used the single-particle level schemes given in Ogle et al. ${ }^{11} \mathrm{~A}$ diagonalization in a space which includes all particle-hole pairs within an energy range of $2 \hbar \omega_{0}$ above and below the Fermi surface is not yet possible with our computing facilities. Therefore the following procedure for selection of pairs was chosen: (i) Any combination within the interval $\epsilon_{p} \pm \hbar \omega_{0}$ is included; (ii) the particle-hole pairs outside this energy interval are included in the order of decreasing absolute value of the single-particle matrix elements. This procedure

TABLE I. Energies and transition probabilities of ${ }^{160} \mathrm{Dy},{ }^{170} \mathrm{Yb}$, and ${ }^{184} \mathrm{~W}$. In general only the most collective state of the low-lying levels of each $K^{\pi}$ combination is given. In the case of the GQR we give the energy of the most collective state and the total strength which generally is distributed over a few levels within an interval of $1 \mathrm{MeV}$.

\begin{tabular}{|c|c|c|c|c|c|c|c|}
\hline Nucleus & $K^{\pi}$ & \multicolumn{2}{|c|}{$\mathrm{E}[\mathrm{MeV}]$} & \multicolumn{2}{|c|}{$B\left(E 2,0^{+}+2^{+}\right) \quad\left[e^{2} \mathrm{fm}^{4}\right]$} & \multicolumn{2}{|c|}{$\begin{array}{c}B(E O)\left[e^{2} \mathrm{fm}^{4}\right] \\
\text { th }\end{array}$} \\
\hline${ }^{160}$ Dy & $\begin{array}{l}0^{+} \\
1^{+} \\
2^{+}\end{array}$ & $\begin{array}{c}1.38 \\
10.5 \\
1.58 \\
11.7 \\
1.27 \\
13.7\end{array}$ & $\begin{array}{l}1.26 \\
0.97\end{array}$ & $\begin{array}{r}354 \\
873 \\
55 \\
1222 \\
468 \\
665\end{array}$ & $\begin{array}{r}220 \pm 70^{\mathrm{a}} \\
1050 \pm 80^{\mathrm{b}}\end{array}$ & $\begin{array}{l}211 \\
921\end{array}$ & \\
\hline $170_{Y b}$ & $\begin{array}{l}0^{+} \\
1^{+} \\
2^{+}\end{array}$ & $\begin{array}{c}1.14 \\
1.32 \\
9.7 \\
2.28 \\
11.9 \\
1.41 \\
13.4\end{array}$ & $\begin{array}{l}1.07 \\
1.23 \\
\\
1.14\end{array}$ & $\begin{array}{r}394 \\
47 \\
1223 \\
46 \\
1067 \\
242 \\
842\end{array}$ & $\begin{array}{l}240 \pm 30^{a} \\
190 \pm 60^{a}\end{array}$ & $\begin{array}{r}202 \\
16 \\
1165\end{array}$ & \\
\hline $184 \mathrm{~W}$ & $\begin{array}{l}0^{+} \\
1^{+} \\
2^{+}\end{array}$ & $\begin{array}{c}1.23 \\
1.40 \\
9.7 \\
1.82 \\
11.4 \\
0.94 \\
1.45 \\
12.6\end{array}$ & $\begin{array}{l}1.00 \\
1.32\end{array}$ & $\begin{array}{r}41 \\
36 \\
1013 \\
98 \\
1316 \\
154 \\
198 \\
938\end{array}$ & $\begin{array}{r}1250 \pm 50^{d} \\
210 \pm 20^{d}\end{array}$ & $\begin{array}{r}42 \\
867\end{array}$ & $1.90 .4^{\mathrm{a}}$ \\
\hline
\end{tabular}

${ }^{a}$ Ref. 12

${ }^{\mathrm{b}}$ Ref. 13 . works well for the $K^{\pi}=0^{+}$case. For the $1^{+}$and $2^{+}$levels the restriction is somewhat more stringent.

It should be mentioned that we obtain spurious $0^{+}$states at zero energy as a result of the consistent use of the particle-particle interaction. ${ }^{10}$ A spurious $1^{+}$state is also reasonably close to zero (e.g., $E_{1^{+}}{ }^{2}=-0.1 \mathrm{MeV}^{2}$ for ${ }^{160} \mathrm{Dy}$ ).

A summary of our results is given in Table I and in Fig. 1. In the table we give in general only the most collective state of the low-lying levels of each $K^{\pi}$ combination. In Fig. 1 the $B(E \lambda)$ values have been summed in intervals of $0.5 \mathrm{MeV}$. The three peaks between 10 and $14 \mathrm{MeV}$ correspond to the $K^{\pi}=0^{+}, 1^{+}$, and $2^{+}$components of the quadrupole vibration. Coinciding with the $K^{\pi}$ $=0^{+}$peak there is also a peak in $B(E 0)$ since the $K^{\pi}=0^{+}$intrinsic states can be populated or depopulated by monopole transitions, too. The distributions for ${ }^{170} \mathrm{Yb}$ and ${ }^{184} \mathrm{~W}$ are very similar. It is important to point out that no effective charges are used. The agreement for $K^{\pi}=0^{+}$is in general better than for $K^{\pi}=2^{+}$. It is the effect of the truncation of the configuration space which makes the interaction in the $2^{+}$case appear to be too weak. A slight renormalization, i.e., increase of the interaction strength, improves the agreement of energy and $B(E 2)$ values with experiment. Therefore our present $B(E 2)$ values of the giant resonances should be taken as lower limits. The centroids of the GQR are in $\mathrm{Dy}, \mathrm{Yb}$, and $\mathrm{W}$ at $11.58,11.44$, and $11.22 \mathrm{MeV}$, respec-

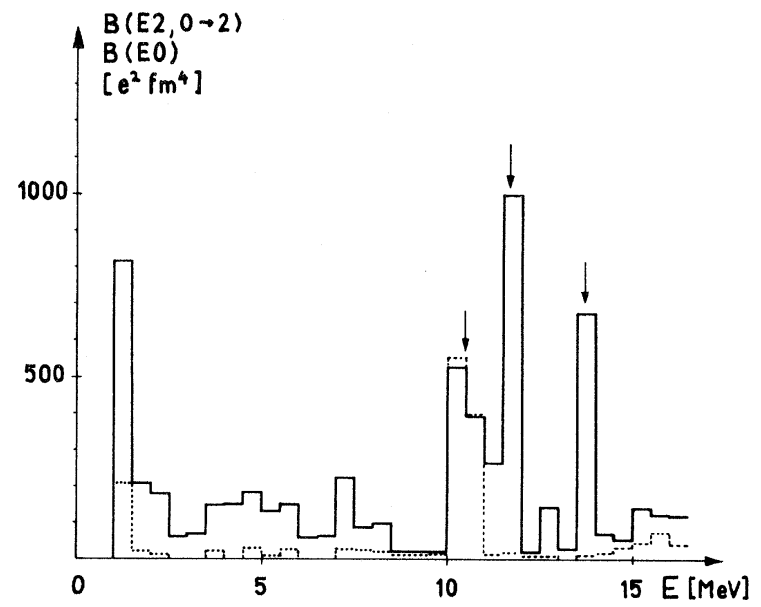

FIG. 1. Distribution of $B(E 2)$ strength (solid line) and $B(E 0)$ strength (dotted line) for ${ }^{160} \mathrm{Dy}$. The $B(E \lambda)$ values have been summed in intervals of $0.5 \mathrm{MeV}$. The exact locations of the resonance maxima are indicated by arrows. 
tively, and the corresponding $B(E 2)$ strengths exhaust the energy-weighted sum rule (EWSR) for isoscalar $E 2$ states to $57 \%, 57 \%$, and $53 \%$, respectively. These values might be compared with the results of several experiments in that region, using hadronic probes, ${ }^{7,8}$ which give the GQR around $12 \mathrm{MeV}$. The experimental $B(E 2)$ strength varies from $80 \%$ up to $150 \%$ of the EWSR. In a $\left(e, e^{\prime}\right)$ experiment ${ }^{6}$ the GQR in ${ }^{150} \mathrm{Nd}$ was found at $11.2 \pm 0.2 \mathrm{MeV}$ and the $B(E 2)$ value exhausts $85 \%$ of the EWSR. If one compares the experimental $B(E 2)$ values with the theoretical results one always has to add the $B(E 0)$ contribution since present experiments do not distinguish between $0^{+}$and $2^{+}$. This gives another $10 \%$ of the EWSR. On the other hand these high-lying (intrinsic) states can be excited also by any $2^{\lambda}$ transition, especially $E 4$. This might be one reason why different experiments give such strongly differing $B(E 2)$ values. To clarify this point one has to perform a distorted-wave Born-approximation analysis similar to that in ${ }^{208} \mathrm{~Pb}$ using microscopic wave functions. ${ }^{16}$

In our model the width of the giant resonances is not calculated. Therefore one has to assume a shape and a width for each line in order to deduce a broadening of the GQR from our results. If we take Gaussian shapes with a full width at half-maximum of $3.9 \mathrm{MeV}$ as measured ${ }^{8}$ in ${ }^{144} \mathrm{Sm}$, we get a broadening of $1.0,2.3$, and $1.4 \mathrm{MeV}$ in $\mathrm{Dy}, \mathrm{Yb}$, and $\mathrm{W}$, respectively. If we take a full width at half-maximum of $2.8 \mathrm{MeV}$ as measured ${ }^{7}$ in ${ }^{142} \mathrm{Nd}$, the corresponding numbers are $1.3,2.9$, and $1.4 \mathrm{MeV}$. These results might be compared with two experimental values: In ${ }^{150} \mathrm{Nd}$ the broadening measured with electrons ${ }^{7}$ is about $2 \mathrm{MeV}$, whereas the broadening in ${ }^{154} \mathrm{Sm}$ measured with $\alpha$ particles is $1 \mathrm{MeV}$.

In order to get more insight into the structure of the GQR in deformed nuclei we calculated the transition density of these levels into the ground state. This corresponds most closely to the density change of a classical vibration at maximum elongation. In Fig. 2 the microscopically calculated transition densities of the high-lying $K^{\pi}=0^{+}$, $1^{+}$, and $2^{+}$states are shown. The $K^{\pi}=0^{+}$pattern looks very similar to a classical $\beta$ vibration. But instead of being small inside the nucleus, the transition density has an appreciable value there as one would expect from a classical breathing mode. Also the $K^{\pi}=1^{+}$and $2^{+}$excitations show the features of high collectivity, i.e., the microscopic transition densities are very similar to a vibrating liquid drop ( $\gamma$ vibration) with little addi-

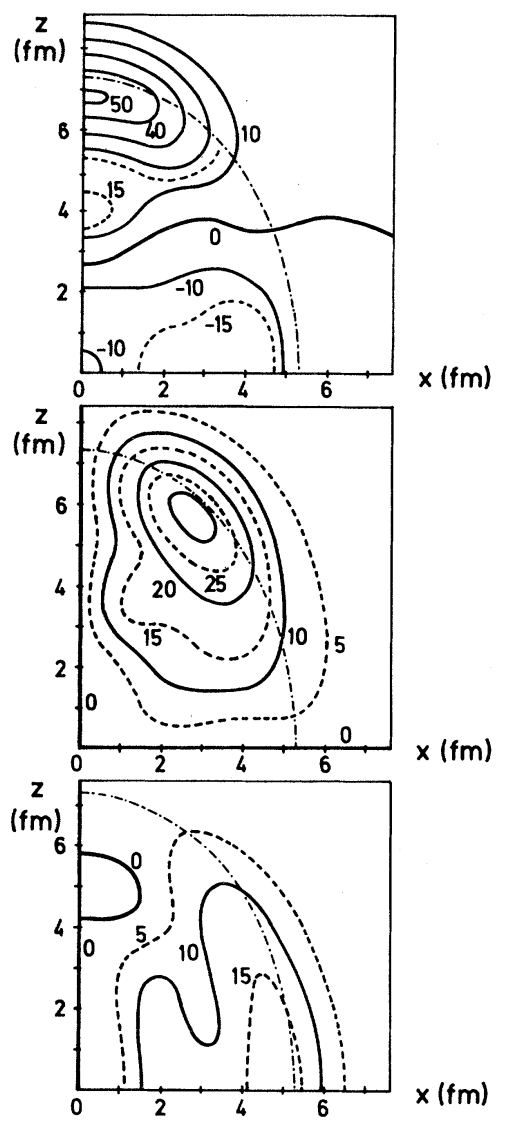

FIG. 2. Microscopically calculated transition densities $\rho_{K}(\overrightarrow{\mathrm{r}})$ of the $K^{\pi}=0^{+}, 1^{+}$, and $2^{+}$giant resonances in ${ }^{160} \mathrm{Dy}$ in units of $10^{-4} \mathrm{fm}^{-3}$. From top to bottom, $K^{\pi}=0^{+}$, $1^{+}$, and $2^{+}$components of the resonance. Since $\rho_{K}$ has the same reflection symmetry and dependence on the angle $\varphi$ as $Y_{2, K}(\theta, \varphi)$, only one quadrant of the $x-z$ plane is shown. The dash-dotted line indicates the boundary of the nucleus where the density has fallen to half its inside value.

tional structure. This behavior is typical for all nuclei investigated so far. The low-lying collective $K^{\pi}=0^{+}$and $2^{+}$states, however, deviate strongly from that classical picture.

${ }^{1}$ R. Pitthan and Th. Walcher, Phys. Lett. 36B, 563 (1971); M. B. Lewis and F. E. Bertrand, Nucl. Phys. A196, 337 (1972); S. Fukuda and Y. Torizuka, Phys. Rev. Lett. 29, 1109 (1972).

${ }^{2}$ G. R. Satchler, Phys. Rep. 14C, 98 (1974).

${ }^{3} \mathrm{~A}$. Bohr and B. R. Mottelson, in Proceedings of the International Symposium on Neutron Capture Gamma Ray Spectroscopy, Studsvik, Sweden, 1969 (International Atomic Energy Agency, Vienna, Austria, 1969).

${ }^{4}$ P. Ring and J. Speth, Phys. Lett. $\underline{44 B}, 477$ (1973), 
and Nucl. Phys. A235, 315 (1974); S. Krewald and J. Speth, Phys. Lett. 52B, 295 (1974).

${ }^{5}$ G. F. Bertsch, Phys. Rev. Lett. 31, 121 (1973); G. F. Bertsch and S. F. Tsai, Phys. Rep. 18C, 126 (1975).

${ }^{6}$ A. Schwierczinski et al., Phys.Lett. 55B, 171 (1975)。

${ }^{7}$ N. Marty et al., Nucl. Phys. A238, $93(1975)$.

${ }^{8}$ T. Kishimoto et al., Phys. Rev. Lett. 35, 552 (1975);

D. H. Youngblood et al., to be published.

${ }^{9}$ R. Ligensa and W. Greiner, Nucl. Phys. A92, 673 (1967).

${ }^{10}$ D. Zawischa and J. Speth, Phys. Lett. 56B, 225
(1975) .

${ }^{11}$ W. Ogle, S. Wahlborn, R. Piepenbring, and S. Fredriksson, Rev. Mod. Phys. 43, 424 (1971).

${ }^{12} \mathrm{P}$. Koldewijn, private communication.

${ }^{13} \mathrm{R}$. N. Oethberg et al., Nucl. Phys. A219, 543 (1974).

${ }^{14}$ D. G. Burk and B. Elbek, K. Dan. Vidensk. Selsk., Mat.-Fys. Medd. 36, No. 6 (1967).

${ }^{15}$ R. C. Greenwood and C. W. Reich, Nucl. Phys. A223, 66 (1974).

${ }^{16}$ E. C. Halbert, J. B. McGrory, G. R. Satchler, and J. Speth, Nucl. Phys. A245, 189 (1975). 